\title{
Dynamics Analysis of the Melt Conveying Process in a Novel Extruder
}

\author{
Zan Huang ${ }^{1,2,3}$ \\ ${ }^{1}$ School of Marine Engineering, Guangzhou Maritime Institute, Guangzhou, Guangdong 510725, China \\ ${ }^{2}$ Department of Chemical Engineering, Hong Kong University of Science and Technology, Clear Water Bay, \\ Kowloon, Hong Kong \\ ${ }^{3}$ Center for Polymer Processing and Systems, HKUST Fok Ying Tung Research Institute, Nansha, Guangzhou, \\ Guangdong 511458, China
}

Correspondence should be addressed to Zan Huang; zhuanggz@aliyun.com

Received 23 August 2015; Revised 27 October 2015; Accepted 27 October 2015

Academic Editor: Matheus Poletto

Copyright (C) 2015 Zan Huang. This is an open access article distributed under the Creative Commons Attribution License, which permits unrestricted use, distribution, and reproduction in any medium, provided the original work is properly cited.

\begin{abstract}
This study investigates dynamics of the melt conveying process in a novel extruder in which a polymer experiences a primarily elongational deformation field. The working principle of the novel extruder is completely different from that of conventional screw extruders. At the last stage of polymer processing in the proposed novel extruder, melt is conveyed through feeding and discharging processes. Here we present a mathematical model to analyze dynamics in the melt conveying process. Melt extrusion is primarily influenced by the position of vane chamber and the structural parameters of the novel extruder such as eccentricity.
\end{abstract}

\section{Introduction}

Polymer manufacturing involves melting and plasticizing material from glass to viscous states. During this process, the solid bed disappears completely and the polymer is fully melted. The role of a metering section is to further plasticize and homogenize the viscous polymer melt. At the end of the metering section, the melt is dispensed from the extruder headpiece at constant pressure and temperature.

Polymer melt flow in the metering section must abide by the laws of conservation of mass, momentum, and energy. To solve the corresponding equations of continuity, motion, and energy, the material parameters of the polymer must be defined by an appropriate constitutive equation. Because polymer melt generally behaves as a non-Newtonian fluid, a complex constitutive equation is required to accurately model the melt behavior. Because of this complexity, it is difficult to obtain an analytical solution for the complex flow field and geometric parameters of the extruder $[1,2]$. To simplify the system, approximations are made which reduce the problem into less complex terms. A linear constitutive model or the equivalent or average Newtonian viscosity has been used to simplify the constitutive equation of the melt
$[3,4]$. Alternatively, the melt flow in the metering section of a screw extruder can be considered as the linear superposition of drag flow and pressure flow by simplifying the flow field [5]. More recently, numerical simulations have been used to understand the melt conveying process [6-12]. In a dynamic polymer extruder, changes in the melt elasticity are more apparent when the flow is disturbed by the action of a vibrational field. Zhang and Qu [13] corrected the Tanner constitutive equation and established a dynamic model of the melt conveying process when exposed to a vibration force field.

In recent years, many scholars have proposed methods to reduce the power consumption of an extruder. For example, $\mathrm{Qu}$ et al. [14-16] introduced the pulsating electromagnetic force field in the extrusion process, which reduced overall power consumption. The novel vane extruder introduced in this study provides a reduction in extruder power consumption, which can be verified by experiments [17]. Qu et al. [18] have also studied the power consumption of the extrusion process in a vane extruder.

In this paper, an analytical model of polymer melt extrusion is established, including solving the velocity distribution, 


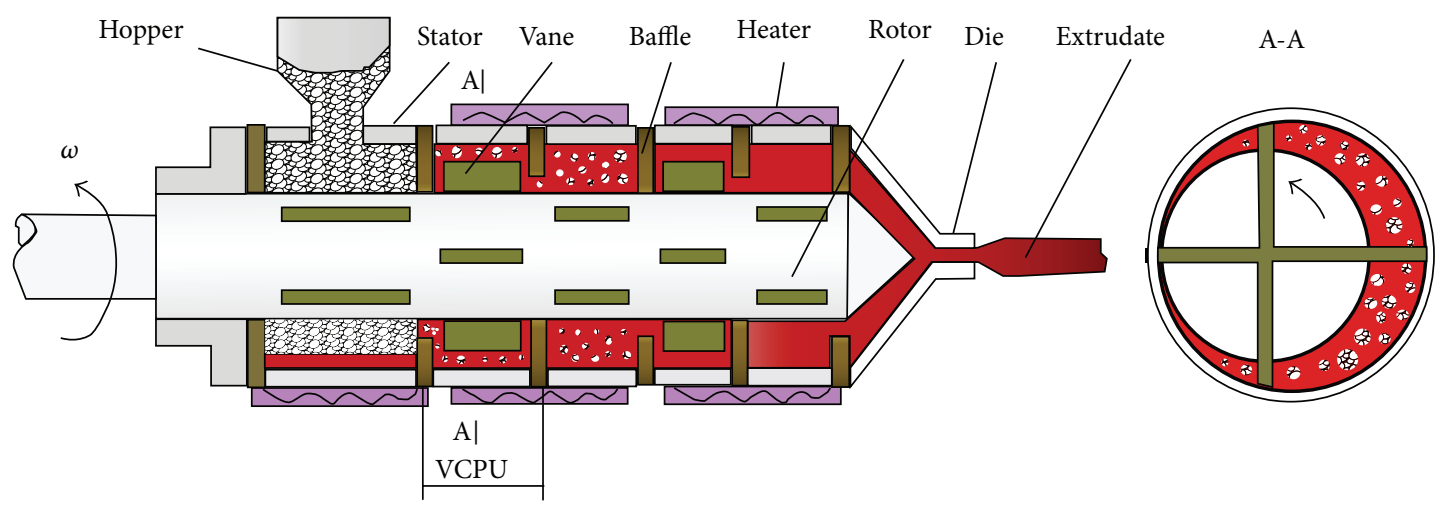

FIgURE 1: The schematic diagram of the novel extruder.

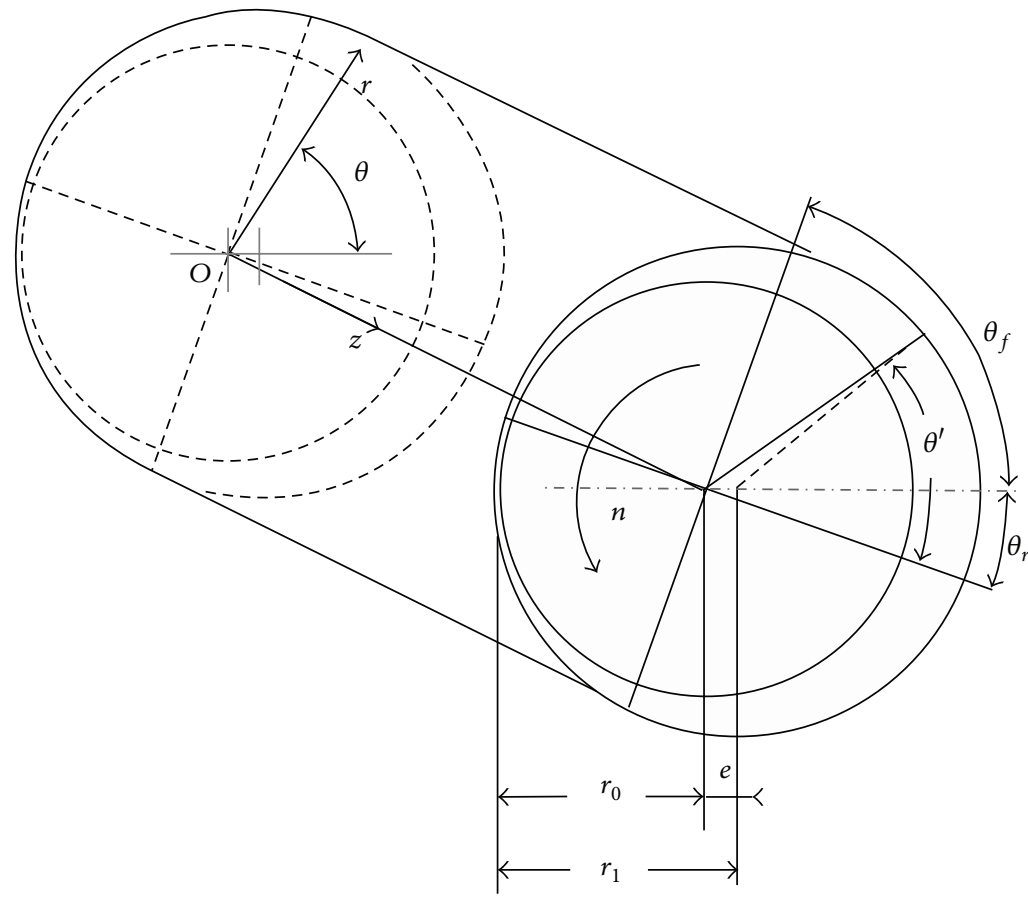

FIgURE 2: The cylindrical coordinate system in VCPU.

pressure gradient, extrusion flow rate, and dynamics of the melt flow field.

\section{Description of the Melt Conveying Process in the Extensional Extruder}

Figure 1 shows the internal structure of a vane extruder. As introduced by Qu et al. [19], a vane extruder includes several Vane Conveying and Plasticizing Units (VCPUs) throughout the chamber. Figure 2 shows the VCPUs in the cylindrical coordinate system $(r, \theta, z)$, where the origin $O$ is defined as the center of the rotor, $r$ gives the radial position along the rotor shaft, $\theta$ is defined along the circumferential direction of the VCPUs where the rotation direction of the rotor is defined as positive, and $z$ is the axial direction of the rotor where the positive direction is defined along the rotor shaft from the feed material baffle to the discharge material baffle. The main geometric parameters of the VCPU system are (1) the outer radius of the rotor $r_{0} ;(2)$ the inner radius of the stator $r_{1} ;(3)$ the axial width of each VCPU $W$; $(4)$ the radial coordinate value of the point of the inner arc of the stator $r_{2}$; (5) the eccentricity $e$ between the center of the rotor $O$ and the center of the stator $O_{1} ;(6)$ the length of the vane $\Delta r_{v} ;(7)$ the angular coordinate of the front vane $\theta_{f} ;(8)$ the angular coordinate of the rear vane $\theta_{r}$.

According to Figure 2 and the cosine theorem, the inner arc of the stator and the length of the vane are related to the VCPU system geometry by

$$
\begin{aligned}
r_{2}(\theta) & =\sqrt{r_{1}^{2}-e^{2} \sin ^{2} \theta}+e \cos \theta, \\
\Delta r_{v}(\theta) & =e+e \cos \theta-\frac{e^{2} \sin ^{2} \theta}{2 r_{1}} .
\end{aligned}
$$




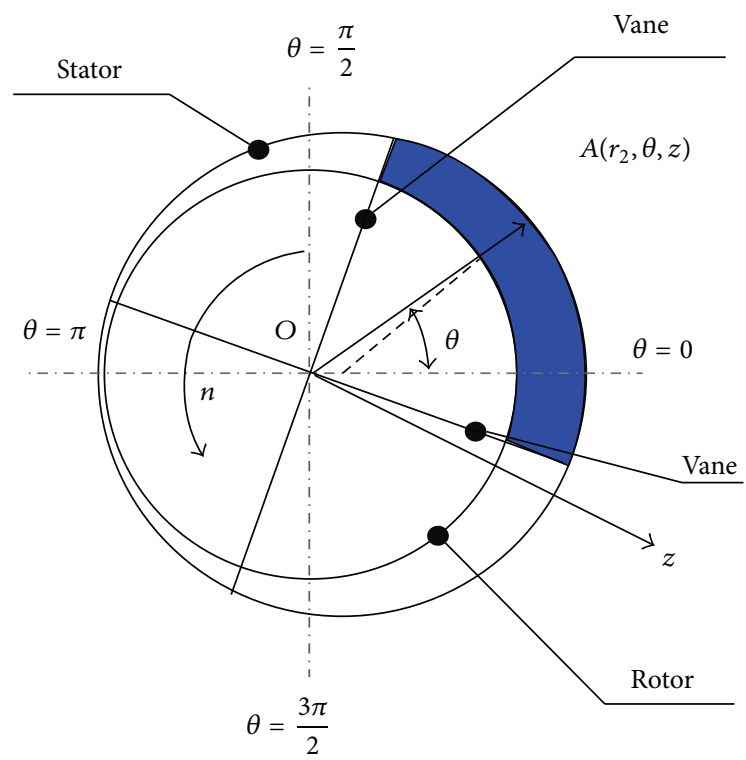

FIGURE 3: The melt conveying model in a chamber of VCPU.

A cross section of the melt conveying zone in the vane extruder is shown in Figure 3. To simplify the melt conveying process and allow for an analytical solution, the following assumptions are made:

(i) The chamber is always filled with polymer melt, even when the volume changes with the rotation of the rotor.

(ii) The melt in the vane chamber is always in isothermal laminar flow.

(iii) The melt in vane chamber is incompressible.

(iv) Leakage and the influence of the thickness of each vane and its geometry are neglected.

(v) The influence of gravity is neglected.

(vi) A Newtonian constitutive equation is used for the melt.

Using the above assumptions, the melt conveying model of the vane extruder can be analytically obtained.

Melt conveyance is examined in the melt-mixing zone of the fully filled chamber of the vane extruder. The Reynolds number was assumed to be much less than unity so that inertial effects are neglected. The governing equations for this situation are shown below.

Continuity Equation. Consider

$$
\frac{1}{r} \frac{\partial v_{\theta}}{\partial \theta}+\frac{\partial v_{z}}{\partial z}=0
$$

where $r, \theta$, and $z$ are cylindrical coordinate components, respectively; $v_{r}, v_{\theta}$, and $v_{z}$ are velocity components of $r$ direction, $\theta$-direction, and $z$-direction.

Constitutive Equation. Consider

$$
\tau=\mu \dot{\gamma},
$$

where $\tau$ is stress tensor; $\mu$ is viscosity coefficient dependent deformation rate; $\dot{\gamma}$ is rate of deformation tensor.

Motion Equations. Consider

$$
\begin{aligned}
\frac{\partial p}{\partial r} & =-\frac{2 \mu}{r^{2}} \frac{\partial v_{\theta}}{\partial \theta} \\
\frac{1}{r} \frac{\partial p}{\partial \theta} & =\mu\left[\frac{\partial}{\partial r}\left(\frac{1}{r} \frac{\partial}{\partial r}\left(r v_{\theta}\right)\right)+\frac{1}{r^{2}} \frac{\partial^{2} v_{\theta}}{\partial \theta^{2}}\right], \\
\frac{\partial p}{\partial z} & =\mu\left[\frac{1}{r} \frac{\partial}{\partial r}\left(r \frac{\partial v_{z}}{\partial r}\right)\right],
\end{aligned}
$$

where $p$ is pressure; $r, \theta$, and $z$ are cylindrical coordinate components, respectively; $v_{r}, v_{\theta}$, and $v_{z}$ are velocity components of $r$-direction, $\theta$-direction, and $z$-direction; $\mu$ is viscosity coefficient dependent deformation rate. Furthermore, the boundary conditions are $v_{z}\left(r_{0}, \pi\right)=0$ and $v_{z}\left(r_{2}, \pi / 4\right)=0$.

Equation (2) can be simplified to

$$
\frac{\partial v_{z}}{\partial z}=-\frac{1}{r} \frac{\partial v_{\theta}}{\partial \theta}
$$

Taking the partial derivative of (7) with respect to $z$ and assuming $\partial v_{\theta} / \partial z=0$ gives

$$
\frac{\partial^{2} v_{z}}{\partial z^{2}}=-\frac{1}{r} \frac{\partial^{2} v_{\theta}}{\partial \theta \partial z}=0
$$

Melt velocity is assumed to vary linearly along the length of the extruder, $\partial v_{z} / \partial z=0$. Thus, $v_{z}$ can be defined as

$$
v_{z}=f_{1}(r, \theta) z+f_{2}(r, \theta),
$$

where $f_{1}$ and $f_{2}$ are only functions of $r$ and $\theta$.

Material enters the vane chamber at the origin of the $z$ axis, $z=0$; thus, the maximum axial velocity of melt occurs at the origin. In contrast, the velocity of the melt at the exit, $z=W$, in the most extreme case can be zero, $v_{z=W}=0$. Substituting this boundary condition into (9) gives $f_{2}(r, \theta)=$ $-f_{1}(r, \theta) W$. At the origin $z=0$, the axial velocity of melt feeding into the vane chamber is $v_{z 0}=f_{2}(r, \theta)=-f_{1}(r, \theta) W$. Thus, (9) can be simplified to

$$
v_{z}=v_{z 0}\left(1-\frac{z}{W}\right)
$$

where $W$ is the axial width of VCPU.

In contrast, the terminal end of the chamber discharges melt, so the axial velocity of melt at the terminal side is maximum, while at the origin, $z=0$, the melt velocity is zero, $v_{z=0}=0$. Substituting this boundary condition into (9), the following expression is obtained: $f_{2}(r, \theta)=0$. The axial velocity of the melt at discharge from the vane chamber is $v_{z 0}=f_{1}(r, \theta) W$. Thus, (9) can be further simplified to

$$
v_{z}=v_{z 0} \frac{z}{W} .
$$


Assuming both sides of (4) and (5) are zero, and $\partial v_{\theta} / \partial z=0$, the following expressions are obtained:

$$
\begin{aligned}
\frac{\partial^{2} p}{\partial z \partial r} & =\frac{\partial}{\partial z}\left(-\frac{2 \mu}{r^{2}} \frac{\partial v_{\theta}}{\partial \theta}\right)=0 \\
\frac{1}{r} \frac{\partial^{2} p}{\partial z \partial \theta} & =\frac{\partial}{\partial z}\left(\mu\left[\frac{\partial}{\partial r}\left(\frac{1}{r} \frac{\partial}{\partial r}\left(r v_{\theta}\right)\right)+\frac{1}{r^{2}} \frac{\partial^{2} v_{\theta}}{\partial \theta^{2}}\right]\right) \\
& =0
\end{aligned}
$$

Examining (12), $\partial p / \partial z$ is independent of $r$ and $\theta$.

Substituting (10) into (6) and rearranging give

$$
\left(1-\frac{z}{W}\right)\left[\frac{1}{r} \frac{\partial}{\partial r}\left(r \frac{\partial v_{z 0}}{\partial r}\right)+\frac{1}{r^{2}} \frac{\partial^{2} v_{z 0}}{\partial \theta^{2}}\right]=\frac{1}{\mu} \frac{\partial p}{\partial z} .
$$

In the geometry of a vane extruder, the circumferential distance is far greater than radial distance, and $v_{z}$ is independent of $\theta$; that is, $\partial v_{z 0} / \partial \theta=0$. To solve for $v_{z 0}$, we first examine the inlet, $z=0$. Equation (13) can be simplified to

$$
\frac{1}{r} \frac{\partial}{\partial r}\left(r \frac{\partial v_{z 0}}{\partial r}\right)=\left.\frac{1}{\mu} \frac{\partial p}{\partial z}\right|_{z=0}
$$

The boundary conditions of (14) are (1) when $r=r_{0}, v_{z 0}=0$ and (2) when $r=r_{2}, v_{z 0}=0$.

Solving (14) gives

$$
v_{z 0}
$$

$$
=\left.\frac{1}{4 \mu}\left[\left(r^{2}-r_{0}^{2}\right)-\left(r_{2}^{2}-r_{0}^{2}\right) \frac{\ln r-\ln r_{0}}{\ln r_{2}-\ln r_{0}}\right] \frac{\partial p}{\partial z}\right|_{z=0},
$$

where $v_{z 0}$ is the axial velocity of the melt at discharge from the vane chamber; $p$ is pressure; $\mu$ is viscosity coefficient dependent deformation rate; $r_{2}$ is the radial coordinate value of the point of the inner arc of the stator; $r_{0}$ is the outer radius of the rotor; $r$ and $z$ are cylindrical coordinate component, respectively.

Substituting (15) into (10), the axial velocity $v_{z}$ of melt flow in the vane chamber at the origin (feed location) is given by

$$
\begin{aligned}
v_{z}= & \frac{1}{4 \mu}\left[\left(r^{2}-r_{0}^{2}\right)-\left(r_{2}^{2}-r_{0}^{2}\right) \frac{\ln r-\ln r_{0}}{\ln r_{2}-\ln r_{0}}\right] \\
& \left.\cdot\left(1-\frac{z}{W}\right) \frac{\partial p}{\partial z}\right|_{z=0},
\end{aligned}
$$

where $W$ is the axial width of VCPU and $\left.(\partial p / \partial z)\right|_{z=0}$ can be obtained using the expression for volumetric flow rate:

$$
Q=\iint v_{z 0} r \mathrm{~d} \theta \mathrm{d} r
$$

where $Q$ is the rate of volume flow, $Q=\mathrm{d} V / \mathrm{d} t ; V$ is the volume of the vane chamber; and $v_{z 0}$ is the velocity of the melt entering the vane chamber.
Substituting (15) into (17) and rearranging give

$$
\begin{aligned}
& \left.\frac{1}{4 \mu} \frac{\partial p}{\partial z}\right|_{z=0} \\
& \cdot \int_{\theta_{r}}^{\theta_{f}} \int_{r_{0}}^{r_{2}}\left[\left(r^{2}-r_{0}^{2}\right)-\left(r_{2}^{2}-r_{0}^{2}\right) \frac{\ln r-\ln r_{0}}{\ln r_{2}-\ln r_{0}}\right] \\
& \cdot r \mathrm{~d} r \mathrm{~d} \theta=Q,
\end{aligned}
$$

where $\mu$ is viscosity coefficient dependent deformation rate; $p$ is pressure; $r$ and $z$ are cylindrical coordinate components, respectively; $\theta_{f}$ is the angular coordinate of front vane; $\theta_{r}$ is the angular coordinate of rear vane; $r_{2}$ is the radial coordinate value of the point of the inner arc of the stator; $r_{0}$ is the outer radius of the rotor; $Q$ is the rate of volume flow.

To simplify, the distance $r_{2}$ is defined as $r_{2} \approx r_{1}+$ $(1 / 2) e\left(\cos \theta_{f}+\cos \theta_{r}\right)$, which can be substituted into (18):

$$
\left.\frac{\partial p}{\partial z}\right|_{z=0}=\frac{4 \mu Q}{k\left(\theta_{r}\right)}
$$

where

$$
\begin{gathered}
k\left(\theta_{r}\right)=\frac{1}{4}\left(\theta_{f}-\theta_{r}\right)\left\{-\left(r_{1}+\frac{1}{2} e\left(\cos \theta_{f}+\cos \theta_{r}\right)\right)^{4}\right. \\
\left.+\frac{\left[\left(r_{1}+(1 / 2) e\left(\cos \theta_{f}+\cos \theta_{r}\right)\right)^{2}-r_{0}^{2}\right]^{2}}{\ln \left(r_{1}+(1 / 2) e\left(\cos \theta_{f}+\cos \theta_{r}\right)\right)-\ln r_{0}}\right\},
\end{gathered}
$$

where $k\left(\theta_{r}\right)$ is the function for simplification; $\theta_{f}$ is the angular coordinate of front vane; $\theta_{r}$ is the angular coordinate of rear vane; $r_{1}$ is the inner radius of the stator; $r_{0}$ is the outer radius of the rotor; $e$ is the eccentricity between the center of the rotor and the center of the stator.

The same steps can be performed on the discharge process in the vane chamber, where the volumetric flow rate of melt discharge can be written as

$$
Q=\iint v_{z 0} r \mathrm{~d} \theta \mathrm{d} r
$$

where $Q$ is the rate of volume flow; $v_{z 0}$ is the axial velocity at the discharge point from the vane chamber; $r$ and $\theta$ are cylindrical coordinate components, respectively.

At discharge, the volume of the chamber continues to shrink, so the volumetric flow rate is negative and the absolute value of $Q$ must be used in (21).

Substituting (11) into (6) and setting $z=W$, the axial velocity of the melt at discharge is given by

$$
\begin{aligned}
& v_{z 0} \\
& \quad=\left.\frac{1}{4 \mu}\left[\left(r^{2}-r_{0}^{2}\right)-\left(r_{2}^{2}-r_{0}^{2}\right) \frac{\ln r-\ln r_{0}}{\ln r_{2}-\ln r_{0}}\right] \frac{\partial p}{\partial z}\right|_{z=W} .
\end{aligned}
$$

Substituting (22) into (21) gives the flow rate $Q$, which can then be substituted into (19). The pressure gradient of the melt flow along the $z$-axis at the discharge location is given by

$$
\left.\frac{\partial p}{\partial z}\right|_{z=W}=-\frac{4 \mu Q}{k\left(\theta_{r}\right)},
$$

where the value of $k\left(\theta_{r}\right)$ is given by $(20)$. 
Because the volume of the vane chamber varies periodically with the rotation of the rotor, there is a large change in chamber volume during the feeding process.

\subsection{Axial Velocity}

2.1.1. Feeding Process $\left(\pi / 2<\theta_{r} \leq 7 \pi / 4\right)$. Substituting (19) into (16), the axial velocity $v_{z}$ of the melt in the vane chamber at the inlet can be given by

$$
\begin{aligned}
v_{z}= & \frac{Q}{k\left(\theta_{r}\right)}\left[\left(r^{2}-r_{0}^{2}\right)-\left(r_{2}^{2}-r_{0}^{2}\right) \frac{\ln r-\ln r_{0}}{\ln r_{2}-\ln r_{0}}\right] \\
& \cdot\left(1-\frac{z}{W}\right),
\end{aligned}
$$

where $r_{2}$ is the radial coordinate of the point on the inner surface of the stator in the cylindrical coordinate system in which the origin is the center of the rotor and $r_{2}=r_{1}+e \cos \theta$. Here, $r_{1}$ is the inner radius of the stator, $e$ is the eccentricity between the rotor and the stator, $r_{0}$ is the outer radius of the rotor, $W$ is the axial width of the vane, and $\theta_{r}$ is the angular coordinate of the rear vane of the VCPU in the cylindrical coordinate system. In addition, $Q$ is the volumetric flow rate of the vane chamber at the position $\theta_{r}$. The value of $Q$ is directly proportional to the speed of the rotor. The coefficient function $k\left(\theta_{r}\right)$ relates to the geometric parameters of the vane chamber and the position of vane.

2.1.2. Discharge Process $\left(-\pi / 4 \leq \theta_{r}<\pi\right)$. Substituting (22) and (23) into (11), the axial velocity $v_{z}$ of melt in the vane chamber at the discharge point is given by

$$
v_{z}=-\frac{Q}{k\left(\theta_{r}\right)}\left[\left(r^{2}-r_{0}^{2}\right)-\left(r_{2}^{2}-r_{0}^{2}\right) \frac{\ln r-\ln r_{0}}{\ln r_{2}-\ln r_{0}}\right] \frac{z}{W} .
$$

According to (24) and (25), the axial velocity $v_{z}$ of melt in the VCPU is directly proportional to the speed of the rotor and is related to the geometric parameters of the vane chamber and the position of vane.

The elongational strain rate along the axis is defined in the cylindrical coordinate system in Figure 2 and can be calculated as

$$
\begin{aligned}
& \dot{\gamma}_{z z}=2 \frac{\partial v_{z}}{\partial z}, \\
& \dot{\gamma}_{z z} \\
& =-\frac{2 Q}{W \cdot k\left(\theta_{r}\right)}\left[\left(r^{2}-r_{0}^{2}\right)-\left(r_{2}^{2}-r_{0}^{2}\right) \frac{\ln r-\ln r_{0}}{\ln r_{2}-\ln r_{0}}\right],
\end{aligned}
$$

where $\dot{\gamma}$ is rate of deformation tensor; $v_{z}$ is the velocity components of $z$-direction; $r$ and $z$ are the cylindrical coordinate components, respectively; $r_{0}$ is the outer radius of the rotor; $r_{2}$ is the radial coordinate value of the point of the inner arc of the stator; $W$ is the axial width of VCPU; $k\left(\theta_{r}\right)$ is the function for simplification; $Q$ is the rate of volume flow.

Note. The values of all parameters are identical to those given in (24) for the feeding process and to those given in (25) for the discharge process.

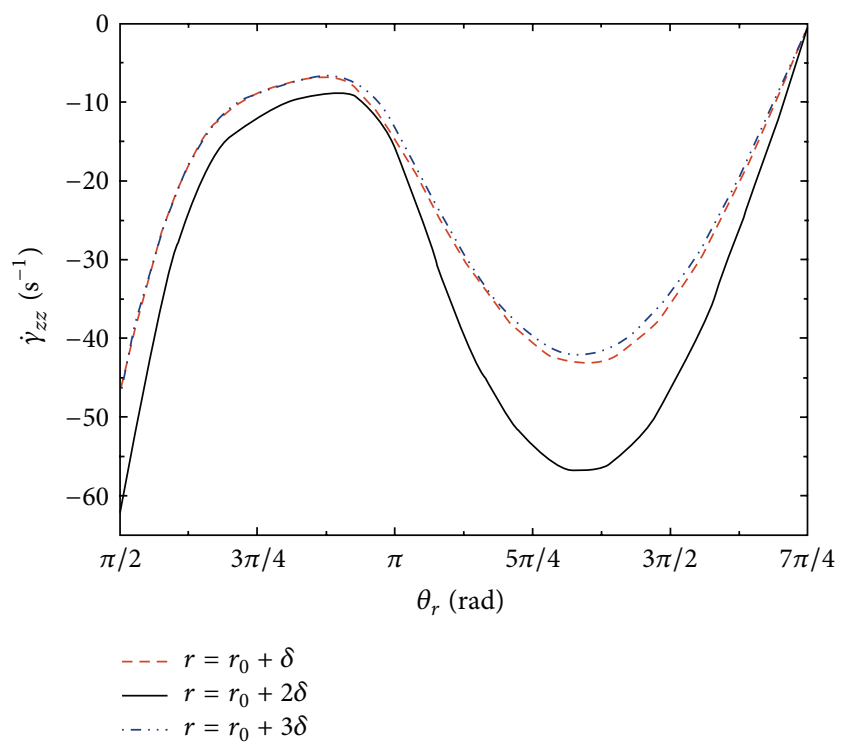

FIGURE 4: The axial tensile strain rate of melt moving in a chamber of VCPU: feed process.

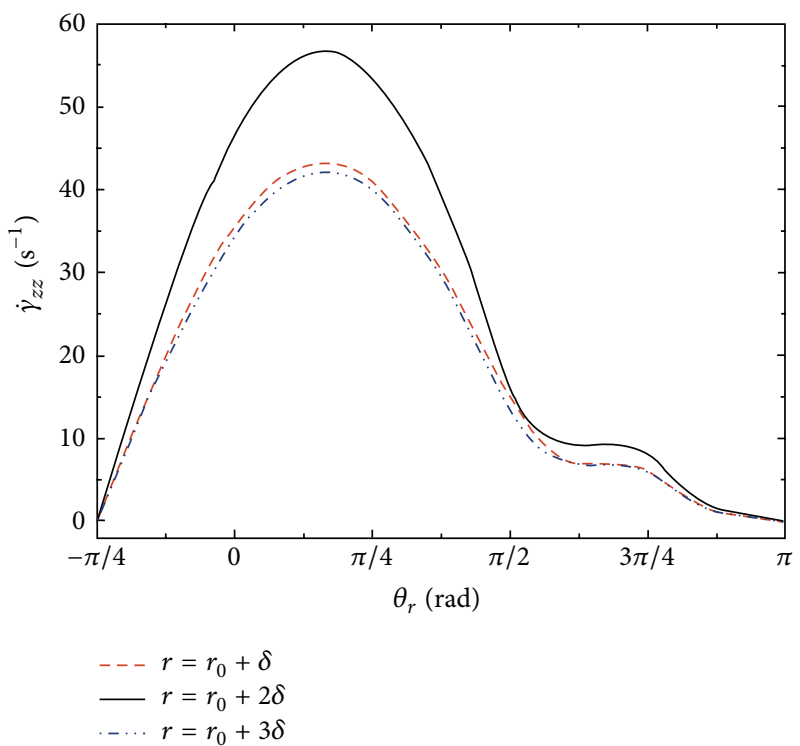

FIGURE 5: The axial tensile strain rate of melt moving in a chamber of VCPU: discharge process.

For example, $r_{0}=20 \mathrm{~mm}, r_{1}=23 \mathrm{~mm}, e=3 \mathrm{~mm}, W=$ $35 \mathrm{~mm}$, and the rotation speed of the rotor $N=60 \mathrm{rpm}$.

The relationship of the axial elongational strain rate of melt moving in the chamber $\dot{\gamma}_{z z}$ and the circumferential position of the rear vane $\theta_{r}$ is shown in Figures 4 and 5.

\section{Mathematical Model}

3.1. Governing Equations. In Figures 4 and 5, the axial rate of strain of the melt $\dot{\gamma}_{z z}<0$ at the inlet, but $\dot{\gamma}_{z z}>0$. In brief, the axial strain rate is at a maximum for a given circumferential position, namely, $r=r_{0}+2 \delta$, and others in inner arc surface 
of stator or in outer arc surface of rotor are smaller; that is, $r=r_{0}+\delta$ or $r=r_{0}+3 \delta$, where $\delta$ is the dimensionless gap.

\subsection{Circumferential Velocity}

3.2.1. Feeding Process. Substituting (24) into continuity (2), the expression can be simplified:

$$
\begin{aligned}
& \frac{\partial v_{\theta}}{\partial \theta} \\
& \quad=\frac{Q \cdot r}{W \cdot k\left(\theta_{r}\right)}\left[\left(r^{2}-r_{0}^{2}\right)-\left(r_{2}^{2}-r_{0}^{2}\right) \frac{\ln r-\ln r_{0}}{\ln r_{2}-\ln r_{0}}\right] .
\end{aligned}
$$

The boundary conditions of melt circumferential velocity are as follows: (1) when $\theta=\theta_{r}, v_{\theta}=\omega r$; (2) when $r=r_{0}$, $v_{\theta}=\omega r_{0}$. Substituting these boundary conditions into (27), the circumferential velocity $v_{\theta}$ can be obtained:

$$
\begin{aligned}
v_{\theta} & =\omega r \\
& +\frac{Q \cdot r}{W \cdot k\left(\theta_{r}\right)}\left[\left(r^{2}-r_{0}^{2}\right)-\left(r_{2}^{2}-r_{0}^{2}\right) \frac{\ln r-\ln r_{0}}{\ln r_{2}-\ln r_{0}}\right] \\
& \cdot\left(\theta-\theta_{r}\right) .
\end{aligned}
$$

3.2.2. Discharge Process. Similarly, substituting (29) into continuity (2), the circumferential velocity $v_{\theta}$ matches the expression given by (28).

\section{Results and Discussion}

4.1. Elongational Strain Rate in the Circumferential Direction. The elongational strain rate in the circumferential direction is defined in the cylindrical coordinate system in Figure 2 and given below:

$$
\begin{aligned}
\dot{\gamma}_{\theta \theta} & =\frac{2}{r} \frac{\partial v_{\theta}}{\partial \theta}, \\
\dot{\gamma}_{\theta \theta} & \\
& =\frac{2 Q}{W \cdot k\left(\theta_{r}\right)}\left[\left(r^{2}-r_{0}^{2}\right)-\left(r_{2}^{2}-r_{0}^{2}\right) \frac{\ln r-\ln r_{0}}{\ln r_{2}-\ln r_{0}}\right] .
\end{aligned}
$$

Note. The values of all parameters are identical to those given in (28) in both the feeding and the discharge processes.

For example, $r_{0}=20 \mathrm{~mm}, r_{1}=23 \mathrm{~mm}, e=3 \mathrm{~mm}, W=$ $35 \mathrm{~mm}$, and the rotational speed of the rotor $N=60 \mathrm{rpm}$.

The relationship between the axial elongational strain rate of melt moving in a chamber of the VCPU $\dot{\gamma}_{\theta \theta}$ and the circumferential position of rear vane $\theta_{r}$ is shown in Figures 6 and 7.

In Figures 6 and 7, the circumferential strain rate of the melt $\dot{\gamma}_{\theta \theta}>0$ at the inlet, but $\dot{\gamma}_{\theta \theta}<0$. In brief, the value of the circumferential strain rate is maximum at any given circumferential position in the vane chamber, namely, $r=$ $r_{0}+2 \delta$, and others in inner arc surface of stator or in outer arc surface of rotor are smaller, namely, $r=r_{0}+\delta$ or $r=r_{0}+3 \delta$.

4.2. Pressure Distribution in the Vane Extruder. In the melt conveying section, the polymer is driven circumferentially

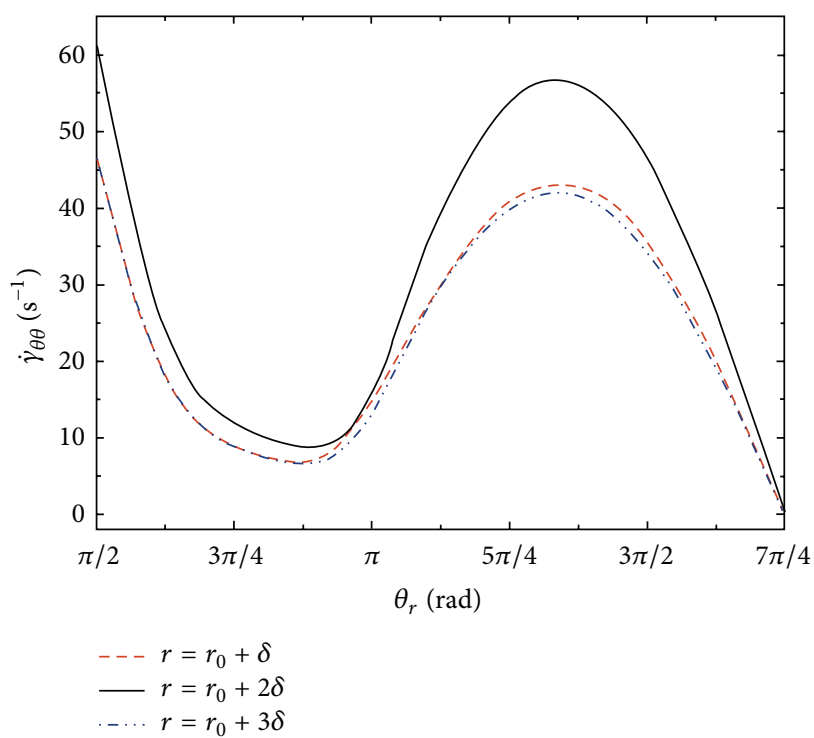

FIGURE 6: The circumferential tensile strain rate of melt moving in a chamber of VCPU: feed process.

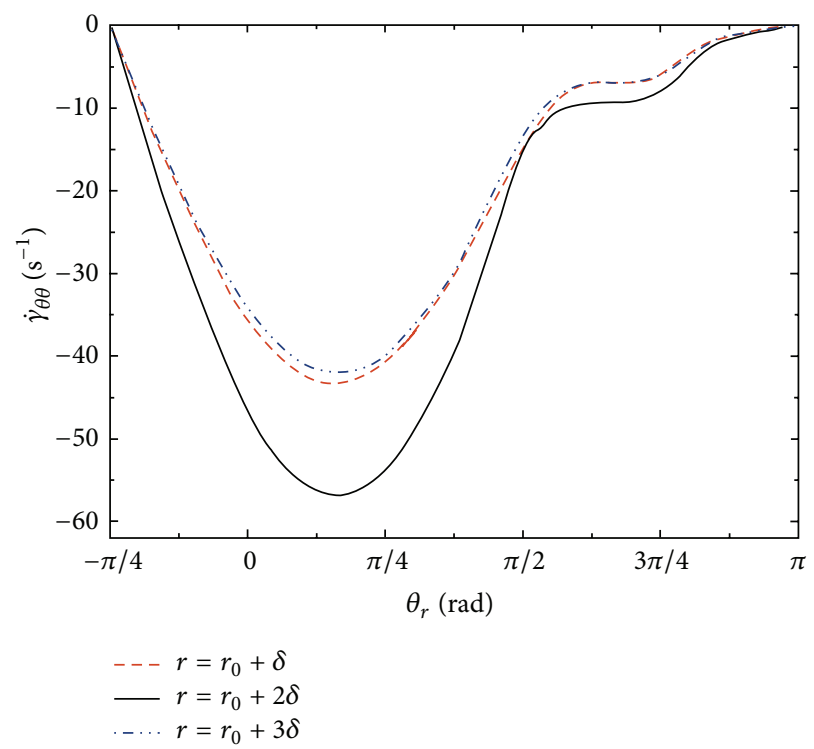

FIGURE 7: The circumferential tensile strain rate of melt moving in a chamber of VCPU: discharge process.

through the VCPU by a rear vane and motion of the rotor. In addition, the VCPU produces positive displacement of the melt, conveying it in the axial direction as the volume of the chamber changes periodically. The axial and circumferential velocity of the melt is different in every position in the vane extruder. Similarly, the axial and circumferential pressure in the melt vary continuously throughout the extruder.

4.2.1. Pressure Distribution in the Axial Direction at the Inlet. Substituting (24) into the motion equation (6) and simplifying give

$$
\frac{\partial p}{\partial z}=\frac{4 \mu Q}{k\left(\theta_{r}\right)}\left(1-\frac{z}{W}\right)
$$


where the values of all variables including $Q$ and $k\left(\theta_{r}\right)$ are identical to those given in (6).

Because the volumetric rate along the axis $Q>0$ and the coefficient function $k\left(\theta_{r}\right)<0, \partial p / \partial z \leq 0$ at the inlet to the vane chamber. This means that the pressure within the vane chamber decreases gradually from the inlet to the outlet of the chamber. As a normal stress field in the axial direction is produced in the vane chamber, melt is conveyed forcibly forward.

The axial pressure difference from the inlet to a point in the vane chamber can be given by

$$
\Delta p_{z}=-\int_{0}^{z} \frac{\partial p}{\partial z} \mathrm{~d} z
$$

where $\Delta p_{z}$ is the pressure difference for $z$-direction.

Substituting (31) into (32), the following expression can be obtained:

$$
\Delta p_{z}=\frac{2 \mu Q}{k\left(\theta_{r}\right)}\left(\frac{z^{2}}{W}-2 z\right) .
$$

With $z=W$, the axial pressure difference from the inlet $(z=$ 0 ) to the outlet is given by

$$
\Delta p_{z}=-\frac{2 \mu Q W}{k\left(\theta_{r}\right)} .
$$

4.2.2. Pressure Distribution in the Circumferential Direction at the Inlet. Substituting (28) into the motion equation (6) and simplifying give

$$
\frac{\partial p}{\partial \theta}=\frac{2 \mu Q}{W \cdot k\left(\theta_{r}\right)}\left[4 r^{2}-\frac{r_{2}^{2}-r_{0}^{2}}{\ln r_{2}-\ln r_{0}}\right]\left(\theta-\theta_{r}\right),
$$

where the values of all variables, including $Q$ and $k\left(\theta_{r}\right)$, are identical to those given in (6).

Because the volumetric axial flow rate $Q>0$ and the coefficient function $k\left(\theta_{r}\right)<0, \theta-\theta_{r} \geq 0,4 r^{2}-\left(r_{2}^{2}-r_{0}^{2}\right) /\left(\ln r_{2}-\right.$ $\left.\ln r_{0}\right)>0$, and $\partial p / \partial \theta \leq 0$ in the entire range $\theta_{r} \leq \theta \leq \theta_{f}$ of the vane chamber. Thus, the circumferential pressure decreases gradually within the vane chamber from rear vane to front vane. The inner arc of the stator gradually moves away from the rotor so that negative pressure is produced in the front of the vane chamber along with the periodic volume expansion of the melt. The circumferential pressure difference from the edge of the chamber $\left(\theta=\theta_{r}\right)$ to a point within the vane chamber is given by

$$
\Delta p_{\theta}=-\int_{\theta_{r}}^{\theta} \frac{\partial p}{\partial \theta} \mathrm{d} \theta
$$

where $\Delta p_{\theta}$ is the pressure difference for $\theta$-direction.

Substituting (35) into (36) and setting $r=\left(r_{0}+r_{2}\right) / 2$ give

$$
\begin{aligned}
& \Delta p_{\theta} \\
& \quad=\frac{\mu Q}{W \cdot k\left(\theta_{r}\right)}\left[\frac{r_{2}^{2}-r_{0}^{2}}{\ln r_{2}-\ln r_{0}}-\left(r_{0}+r_{2}\right)^{2}\right]\left(\theta-\theta_{r}\right)^{2} .
\end{aligned}
$$

At $\theta=\theta_{f}$, the circumferential pressure difference from the extreme side $\left(\theta=\theta_{r}\right)$ to the front side is given by

$$
\begin{aligned}
& \Delta p_{\theta} \\
& =\frac{\mu Q}{W \cdot k\left(\theta_{r}\right)}\left[\frac{r_{2}^{2}-r_{0}^{2}}{\ln r_{2}-\ln r_{0}}-\left(r_{0}+r_{2}\right)^{2}\right]\left(\theta_{f}-\theta_{r}\right)^{2},
\end{aligned}
$$

where $\theta_{f}$ and $\theta_{r}$ are the most front and the most rear circumferential coordinates, respectively. When $\pi \leq \theta_{r} \leq$ $7 \pi / 4, \theta_{f}-\theta_{r}=\pi / 2$; when $\pi / 2<\theta_{r}<\pi, \theta_{f}-\theta_{r}=\theta_{r}-\pi / 2$.

Synthesizing (30) and (37), the characteristic pressure function at the inlet is given by

$$
\begin{aligned}
p=p_{i} & +\frac{2 \mu Q}{k\left(\theta_{r}\right)}\left(2 z-\frac{z^{2}}{W}\right) \\
+ & \frac{\mu Q}{W \cdot k\left(\theta_{r}\right)}\left[\left(r_{0}+r_{2}\right)^{2}-\frac{r_{2}^{2}-r_{0}^{2}}{\ln r_{2}-\ln r_{0}}\right] \\
& \cdot\left(\theta-\theta_{r}\right)^{2},
\end{aligned}
$$

where $p_{i}$ is the pressure at the edge of the vane chamber $(z=$ $\left.0, \theta=\theta_{r}\right)$.

A gap is produced due to the volume enlargement at the front vane in the vane chamber, and the final side of the chamber can be filled with melt. The pressure at the front of the vane in the vane chamber is therefore roughly equal to atmospheric pressure; that is, $p\left(\theta_{f}, W\right)=p_{0}$. Substituting into (36) gives

$$
\begin{aligned}
p_{i}= & p_{0}-\frac{2 \mu Q W}{k\left(\theta_{r}\right)} \\
& -\frac{\mu Q \pi^{2}}{4 W \cdot k\left(\theta_{r}\right)}\left[\left(r_{0}+r_{2}\right)^{2}-\frac{r_{2}^{2}-r_{0}^{2}}{\ln r_{2}-\ln r_{0}}\right],
\end{aligned}
$$

where $p_{i}$ is the pressure at the final side of the vane chamber; $p_{0}$ is the atmospheric pressure; $\mu$ is viscosity coefficient dependent deformation rate; $Q$ is the rate of volume flow; $W$ is the axial width of VCPU; $k\left(\theta_{r}\right)$ is the function for simplification; $r_{0}$ is the outer radius of the rotor; $r_{2}$ is the radial coordinate value of the point of the inner arc of the stator.

4.2.3. Pressure Distribution in the Axial Direction at the Outlet. Substituting (25) into the motion equation (6) and simplifying give

$$
\frac{\partial p}{\partial z}=-\frac{4 z \mu Q}{W \cdot k\left(\theta_{r}\right)} .
$$

Substituting (41) into (32) gives

$$
\Delta p_{z}=\frac{2 \mu Q z^{2}}{W \cdot k\left(\theta_{r}\right)} .
$$

At $z=W$, the axial pressure difference from the inlet to the outlet is given by

$$
\Delta p_{z}=\frac{2 \mu Q W}{k\left(\theta_{r}\right)}
$$


4.2.4. Pressure Distribution in the Circumferential Direction at the Outlet. The circumferential pressure differential expression is identical to (37).

Combining (43) and (37), the characteristic pressure function at the outlet of the vane chamber is given by

$$
\begin{aligned}
p= & p_{0}+\frac{2 \mu Q(z-W)^{2}}{W \cdot k\left(\theta_{r}\right)} \\
- & \frac{\mu Q}{W \cdot k\left(\theta_{r}\right)}\left[\left(r_{0}+r_{2}\right)^{2}-\frac{r_{2}^{2}-r_{0}^{2}}{\ln r_{2}-\ln r_{0}}\right] \\
& \cdot\left(\theta-\theta_{f}\right)^{2},
\end{aligned}
$$

where $p$ is the pressure; $p_{0}$ is the atmospheric pressure.

\section{Conclusions}

The above analysis supports a number of conclusions:

(i) The polymer melt conveying process in the vane extruder is completely different from the process in conventional screw extruders.

(ii) The pressure within the chamber is influenced by the location and volume within the chamber.

(iii) The dynamics of melt conveyance in a vane chamber depend mostly on pressure to overcome viscous forces. Therefore, the power consumption of a vane extruder increases with pressure and viscosity within the chamber and is directly proportional to the speed of the rotor.

\section{Nomenclatures}

$r, \theta, z: \quad$ Cylindrical coordinate, $\mathrm{mm}, \mathrm{mm}, \mathrm{mm}$

$O: \quad$ The origin of the cylindrical coordinate system, that is, the center of the rotor

$\mathrm{O}_{1}$ : The center of the stator

$r_{0}$ : The outer radius of the rotor, $\mathrm{mm}$

$r_{1}$ : The inner radius of the stator, $\mathrm{mm}$

$W$ : The axial width of VCPU, mm

$r_{2}$ : The radial coordinate value of the point of the inner arc of the stator, $\mathrm{mm}$

$e: \quad$ The eccentricity between the center of the rotor and the center of the stator, $\mathrm{mm}$

$\Delta r_{v}: \quad$ The extend length of vane, $\mathrm{mm}$

$\theta_{f}: \quad$ The angular coordinate of front vane, $\mathrm{rad}$

$\theta_{r}$ : The angular coordinate of rear vane, $\mathrm{rad}$

$\tau: \quad$ Stress tensor, $\mathrm{N} / \mathrm{cm}^{2}$

$\mu$ : Viscosity coefficient dependent deformation rate

$\dot{\gamma}: \quad$ Rate of deformation tensor, $\mathrm{s}^{-1}$

$p: \quad$ Pressure, $\mathrm{N} / \mathrm{cm}^{2}$

$v_{r}, v_{\theta}, v_{z}$ : Velocity components of $r$-direction, $\theta$-direction, and $z$-direction, $\mathrm{cm} / \mathrm{s}$

$f_{1}, f_{2}$ : Arbitrary functions for $r$ and $\theta$

$Q: \quad$ The rate of volume flow
$V: \quad$ The volume of vane chamber

$v_{z 0}$ : The velocity of feeding melt at the inlet of the

vane chamber

$k\left(\theta_{r}\right): \quad$ The function for simplification

$N: \quad$ The rotational speed of the rotor, rpm

$\delta: \quad$ The dimensionless gap

$\Delta p_{\theta}, \Delta p_{z}$ : The pressure difference for $\theta$-direction and $z$-direction

$p_{i}: \quad$ The pressure at the final side of the vane

$p_{0}: \quad$ The atmospheric pressure.

\section{Conflict of Interests}

The author declares that there is no conflict of interests regarding the publication of this paper.

\section{Acknowledgments}

The author wishes to acknowledge the Guangzhou Science and Technology Plan Project (201541) and the "Innovation and Strengthen University" Project of Guangzhou Maritime Institute (A330106, B510647) for financial support.

\section{References}

[1] B. Yang and L. J. Lee, "Process control of profile extrusion using thermal method. Part I: mathematical modeling and system analysis," Polymer Engineering \& Science, vol. 28, no. 11, pp. 697707, 1988 .

[2] B. Yang and L. J. Lee, "Process control of profile extrusion using thermal method. Part II: closed loop control," Polymer Engineering Science, vol. 28, no. 11, pp. 708-717, 1988.

[3] E. Broyer, C. Gutfinger, and Z. Tadmor, "Evaluating flows of non-Newtonian fluids by the method of equivalent Newtonian viscosity," AIChE Journal, vol. 21, no. 1, pp. 198-200, 1975.

[4] M. V. Bruschke and S. G. Advani, "Flow of generalized Newtonian fluids across a periodic array of cylinders," Journal of Rheology, vol. 37, no. 3, p. 479, 1993.

[5] F. W. Kroesser and S. Middleman, "The calculation of screw characteristics for the extrusion of non-Newtonian melts," Polymer Engineering \& Science, vol. 5, no. 4, pp. 230-234, 1965.

[6] R. M. Griffith, "Fully developed flow in screw extruders. Theoretical and experimental study," Industrial \& Engineering Chemistry Fundamentals, vol. 1, no. 3, pp. 180-187, 1962.

[7] H. J. Zamodits, "Flow of polymer melts in extruders. Part I. the effect of transverse flow and of a superposed steady temperature profile," Journal of Rheology, vol. 13, no. 3, p. 357, 1969.

[8] D. F. Dyer, "A numerical solution for the single-screw extrusion of a polymer melt," AIChE Journal, vol. 15, no. 6, pp. 823-828, 1969.

[9] E. E. Agur and J. Vlachopoulos, "Numerical simulation of a single-screw plasticating extruder," Polymer Engineering \& Science, vol. 22, no. 17, pp. 1084-1094, 1982.

[10] C. D. Han, K. Y. Lee, and N. C. Wheeler, "An experimental study on plasticating single-screw extrusion," Polymer Engineering \& Science, vol. 30, no. 24, pp. 1557-1567, 1990.

[11] J. Brandao, E. Spieth, and C. Lekakou, "Extrusion of polypropylene. Part I. Melt rheology," Polymer Engineering and Science, vol. 36, no. 1, pp. 49-55, 1996. 
[12] C. Lekakou and J. Brandao, "Extrusion of polypropylene. Part II: process analysis of the metering zone," Polymer Engineering \& Science, vol. 36, no. 1, pp. 56-64, 1996.

[13] J. Zhang and J.-P. Qu, "Nonlinear viscoelasticity of polymer melts in electromagnetic vibration force field," Nonlinear Analysis, Theory, Methods \& Applications, vol. 63, no. 5-7, pp. e977e984, 2005.

[14] J. P. Qu, "Study on the pulsating extrusion characteristics of polymer melt through round-sectioned die," Polymer-Plastics Technology and Engineering, vol. 41, no. 1, pp. 115-132, 2002.

[15] W. Quan, J.-P. Qu, and H. Liang, "Effect of vibration parameters of electromagnetic dynamic plastics injection molding machine on mechanical properties of polypropylene samples," Journal of Applied Polymer Science, vol. 102, no. 2, pp. 972-976, 2006.

[16] G. S. Zeng, C. Xu, Y. J. Liu, and J. Qu, "Hysteresis heat build-up and low temperature processing of polymer under the vibration force field," Advanced Materials Research, vol. 415-417, pp. 200204, 2012.

[17] J.-P. Qu, Z.-T. Yang, X.-C. Yin, H.-Z. He, and Y.-H. Feng, "Characteristics study of polymer melt conveying capacity in vane plasticization extruder," Polymer-Plastics Technology and Engineering, vol. 48, no. 12, pp. 1269-1274, 2009.

[18] J.-P. Qu, X.-Q. Zhao, J.-B. Li, and S.-Q. Cai, “Power consumption in the compacting process of polymer particulate solids in a vane extruder," Journal of Applied Polymer Science, vol. 127, no. 5, pp. 3923-3932, 2013.

[19] J.-P. Qu, G.-Z. Zhang, H.-Z. Chen, X.-C. Yin, and H.-Z. He, "Solid conveying in vane extruder for polymer processing: effects on pressure establishment," Polymer Engineering \& Science, vol. 52, no. 10, pp. 2147-2156, 2012. 

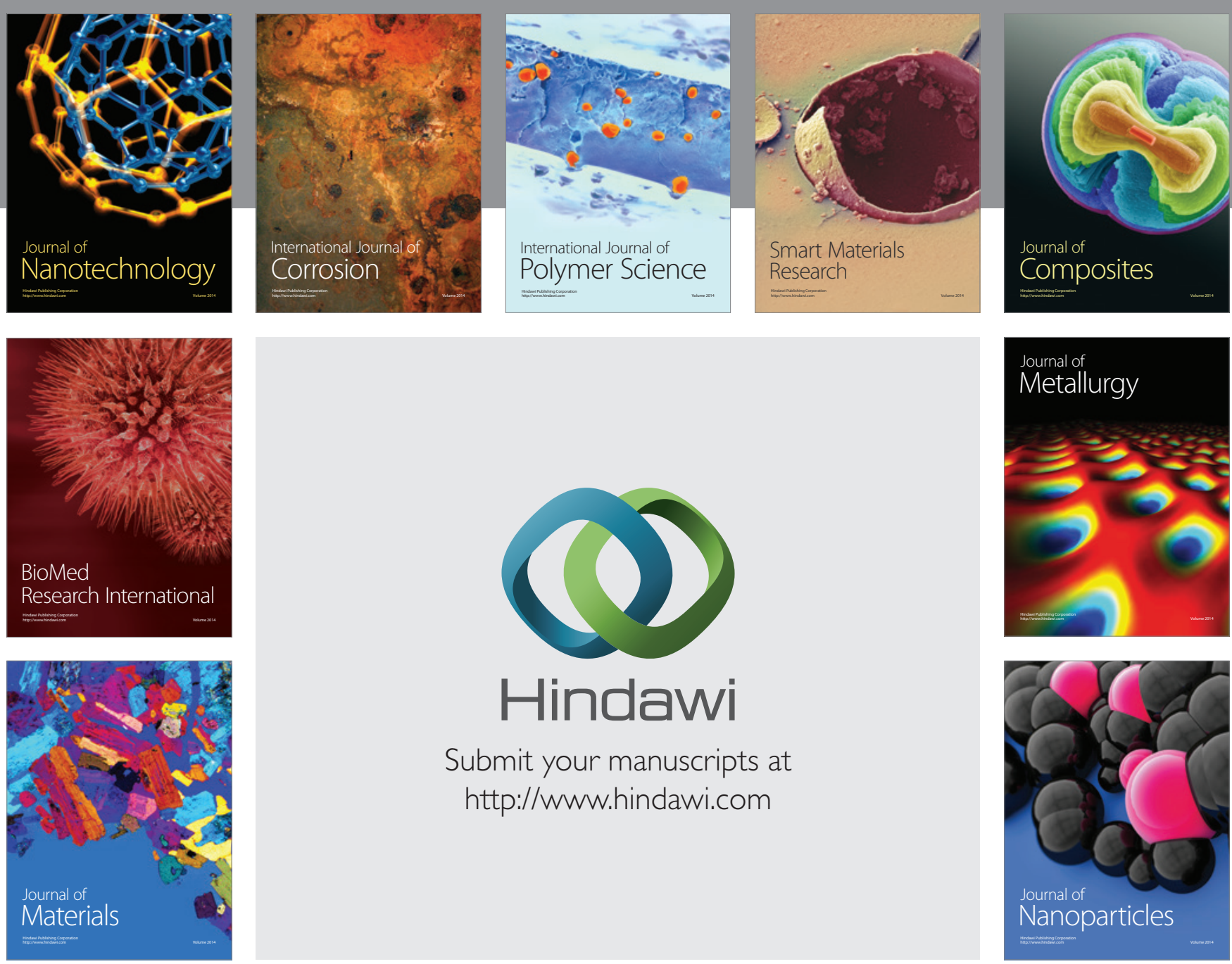

Submit your manuscripts at http://www.hindawi.com
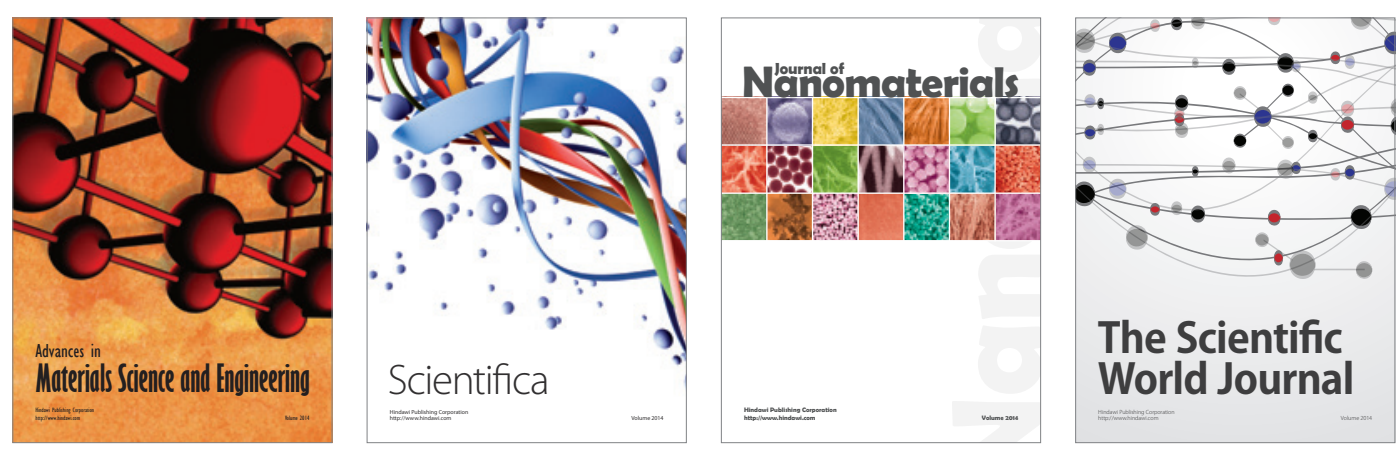

\section{The Scientific World Journal}
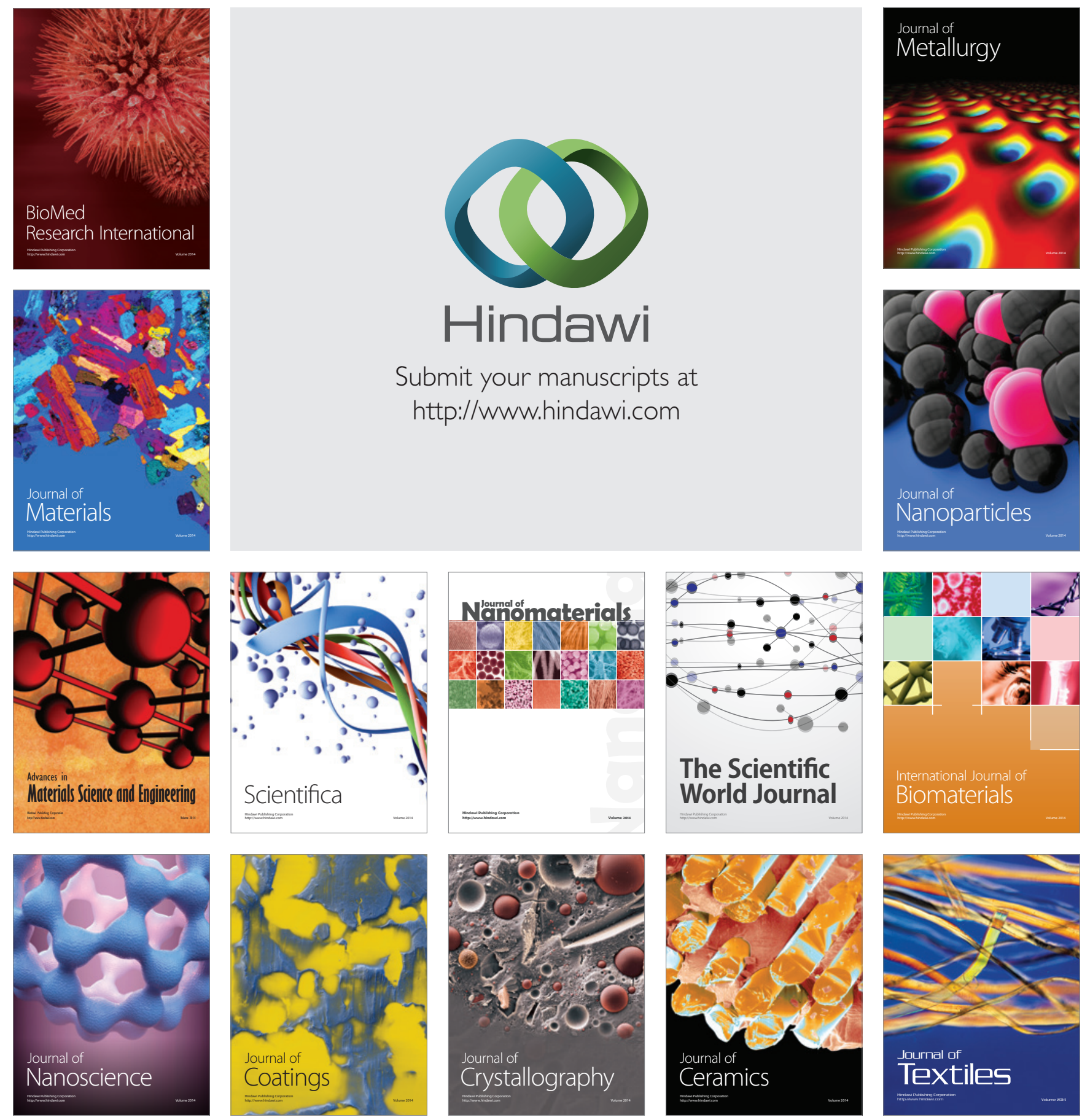